\title{
Endometrioid Carcinoma and Ectopic Pregnancy Coexisting in an Ovarian Endometrioma
}

\author{
${ }^{1}$ Sabahat Rasool, ${ }^{2}$ Tamkin Rabbani, ${ }^{3}$ Omar S Akhtar, ${ }^{4}$ Rana K Sherwani \\ ${ }^{1}$ Senior Resident, Department of Obstetrics and Gynecology, JN Medical College, Aligarh Muslim University, Aligarh, Uttar \\ Pradesh, India \\ ${ }^{2}$ Reader, Department of Obstetrics and Gynecology, JN Medical College, Aligarh Muslim University, Aligarh, Uttar Pradesh \\ India \\ ${ }^{3}$ Senior Resident, Department of Surgery, JN Medical College, Aligarh Muslim University, Aligarh, Uttar Pradesh, India \\ ${ }^{4}$ Professor, Department of Pathology, JN Medical College, Aligarh Muslim University, Aligarh, Uttar Pradesh, India \\ Correspondence: Sabahat Rasool, Senior Resident, Department of Obstetrics and Gynecology, JN Medical College, Aligarh \\ Muslim University, S-5, Azeem Estate, Sir Syed Nagar, Aligarh-202001, Uttar Pradesh, India, Mob: +91-9759051122 \\ +91-9759061122, e-mail: sabahatrasool@yahoo.co.in
}

\begin{abstract}
A 28 years old female presented to our OPD with the complaints of inability to conceive for the last 13 years and a lump in abdomen for the past month. Perspeculum examination revealed a healthy cervix and vagina and a per-vaginum examination revealed a uterus that was normal in size but a lump was palpable in the right adnexa. There was no cervical motion tenderness. Routine investigations were normal. Ultrasonography showed a complex right-sided adnexal mass of 165 by $78 \mathrm{~mm}$ with heterogenous echotexture. Tumor markers were within normal range except serum B-hCG, which was raised. Urine pregnancy test was positive. CT scan reported a welldefined large solid-cystic moderately enhancing pelvic mass with internal septations, seemingly arising from right adnexa. The patient was taken up for a staging laparotomy. Peroperatively, the picture was suggestive of a chronic ectopic pregnancy. Histopathological analysis from the lesion showed an ovarian endometrioid carcinoma and ectopic pregnancy coexisting in the endometrioma complete surgical staging was done, followed by chemotherapy. The patient has responded well to the treatment.
\end{abstract}

Keywords: Endometrioid carcinoma, ectopic pregnancy, coexisting, ovarian endometrioma.

\section{INTRODUCTION}

Coexistence of an ovarian tumor with an ectopic ovarian pregnancy has been rarely reported. We present here a case of an ovarian ectopic gestation coexisting with an ipsilateral ovarian endometrioid carcinoma. To our knowledge, this is the first such case reported of this association.

\section{CASE PRESENTATION}

A 28 years old nulligravid female presented with inability to conceive for thirteen years and a painless lower abdominal lump for one month. The lump had grown in size but was not associated with any other symptoms. Her menstrual cycles were normal with a cycle of 30 days and normal flow for 5 days. However, four months back, she had amenorrhea lasting for two and a half months, followed by spontaneous menstruation approximately 25 days prior to reporting to our hospital. She denied any past or family history of tuberculosis or diabetes.

On examination, she was of average built and nutrition, and was hemodynamically stable. On abdominal examination, there was palpable suprapubic abdominal lump 8 by $8 \mathrm{~cm}$ in size, apparently arising from the pelvis, extending into the right iliac fossa. It was firm-hard in consistency, with a smooth surface and was not freely mobile. There was no palpable organomegaly and no clinically detected free fluid in abdomen. On perspeculum examination, cervix and vagina were healthy and there was no bleeding. On per-vaginum examination, uterus was bulky and a firm to hard right adnexal lump of about $15 \mathrm{~cm}$ was palpable. Cervical excitation was negative and the left adnexa was free. Per-rectal examination revealed a hard lump anteriorly, upper limit of which could not be reached; the rectal mucosa was free.

With a provisional clinical diagnosis of a solid-cystic adnexal mass, she was investigated further.

Her hemoglobin was $10 \mathrm{gm} \%$. Her blood picture, renal and liver function tests and chest X-ray were normal.

Transabdominal ultrasound scan revealed a complex right adnexal mass of 165 by $78 \mathrm{~mm}$, with heterogenous echotexture (Fig. 1). CT scan of the abdomen and pelvis showed a welldefined solid-cystic moderately-enhancing pelvic mass with internal septations, seemingly arising from right adnexa (Fig. 2). 


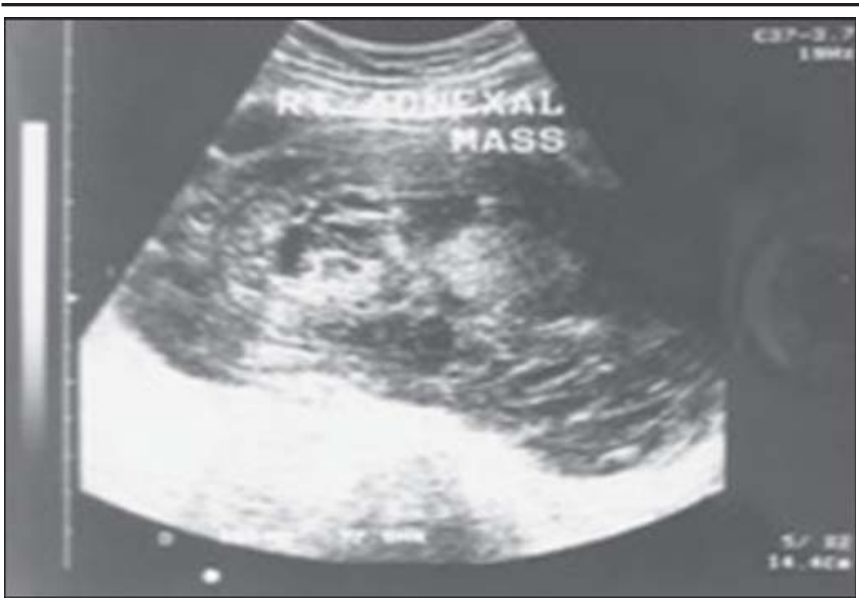

Fig. 1: USG of the patient showing a complex adnexal mass

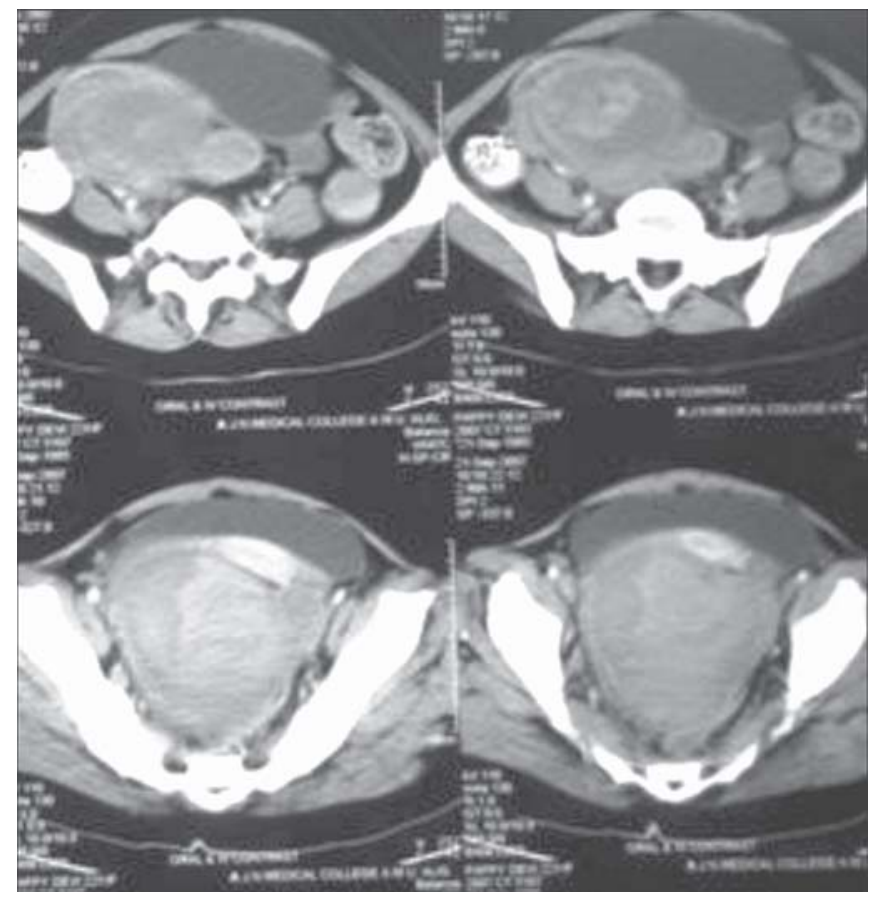

Fig. 2: CECT scan of the pelvis showing a well-defined large solidcystic moderately enhancing pelvic mass with internal septations, seemingly arising from right adnexa

CA-125 and $\alpha$ FP were both within normal range. Serum $\beta$ hCG was $336 \mathrm{mIU}$ per $\mathrm{ml}$ (normal range 0 to $10 \mathrm{mIU} / \mathrm{ml}$ ) and urine pregnancy test performed at the time was positive.

Patient was taken up for staging laparotomy with the likely diagnosis of a germ cell tumor.

Peroperatively a large pelvic hematocele of about 10 by 12 cm was seen on right side, also occupying the pouch of Douglas (Fig. 3). The right ovary could not be separately visualized. A left sided hematosalpinx was also present, however the left ovary and uterus were normal. A 1 by $2 \mathrm{~cm}$ omental nodule was seen. No other abnormality was found. Peritoneal washings

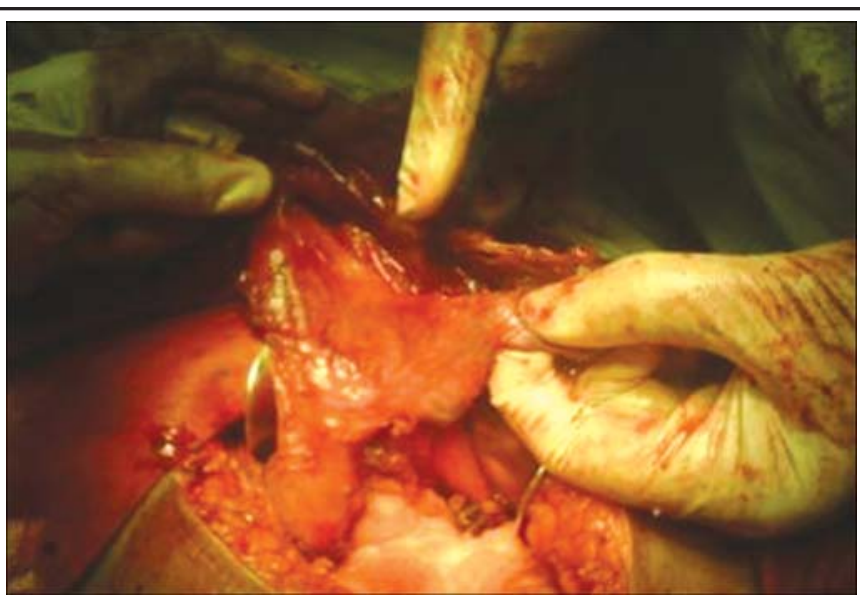

Fig. 3: Intraoperative photograph of the mass after evacuation of its contents, mainly blood clots

were taken. With the likely diagnosis of chronic ectopic pregnancy and hematocele, the staging laparotomy was abandoned and the mass was excised in totality.

Cytological analysis of the peritoneal washings revealed suspicious malignant cells. Histopathological examination of the contents of the hematocele revealed clots, hyalinized and degenerating chorionic villi, all findings consistent with ectopic pregnancy (Fig. 4). Histopathological examination of the wall of the mass revealed endometriosis as well as moderatelydifferentiated malignant glandular structures within ovarian stroma, having endometrioid features (Fig. 5). Peritoneal implant biopsy showed metastatic deposits with areas of necrosis.

With a final diagnosis of endometrioid carcinoma stage IIIC coexistent with an ovarian ectopic gestation, the patient was taken up for hysterectomy, adnexectomy and omentectomy two weeks later. No further pathology was found on the histopathological examination of the excised organs. Her postoperative

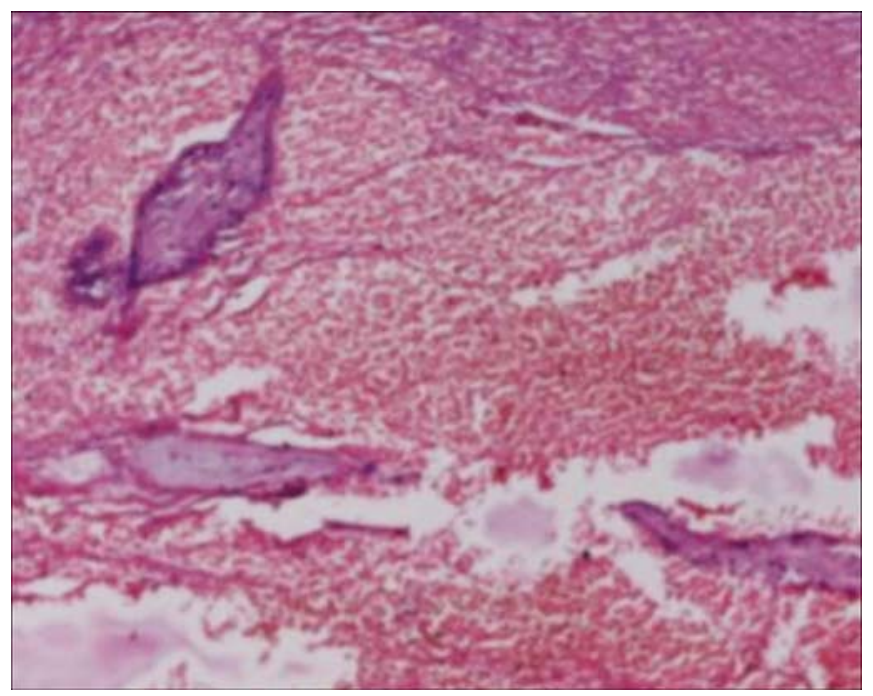

Fig. 4: HPE of the cyst material showing blood clots, hemorrhage, hyalinised and degenerating chorionic villi suggesting ectopic pregnancy 


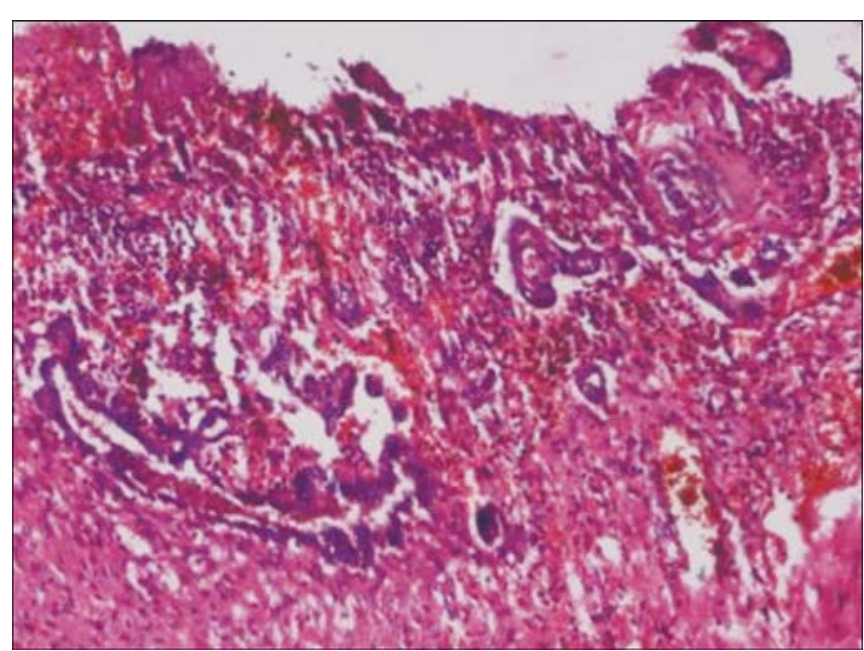

Fig. 5: HPE of the cyst wall showing malignant glandular structures within the ovarian stroma having endometrioid features

recovery was uncomplicated. The patient received four cycles of cisplatin-based chemotherapy. She was doing well when last seen.

\section{DISCUSSION}

Our patient is probably the first reported case of an endometrioid ovarian carcinoma with ipsilateral ectopic pregnancy existing in an ovarian endometrioma.

Endometriosis is the presence of endometrial tissue outside the uterus, the most frequent sites being pelvic viscera and the peritoneum. Its prevalence in patients with infertility ranges between 20 and $90 \%{ }^{1,2}$ Our patient had been infertile for 13 years but other symptoms like dysmenorrhea and dyspareunia were absent. According to some studies, endometriosis increases the risk of developing ovarian cancer. ${ }^{3,4}$ However, this risk is unrelated to the parity of the women with endometriosis. ${ }^{5}$ Endometriosis can lead to pelvic and tubal adhesions and abnormal tubal function, which predisposes to ectopic pregnancy. ${ }^{6}$

Ovarian ectopic pregnancy constitutes $0.5 \%$ of ectopic pregnancies, the risk factors being tubal disease, infertility treatment and intrauterine devices. In our patient, it seems likely that endometriosis led to neoplastic transformation as well as ectopic gestation.

Regarding the management of epithelial ovarian cancers, a complete surgical staging should be done. However, in early low risk disease, fertility sparing surgeries may be considered in young patients. In this case, the disease was advanced, with omental metastasis, and we had to proceed with total abdominal hysterectomy, bilateral salpingo-oophorectomy and omentectomy. The patient also received four-cycles of cisplatin based chemotherapy after surgery.

\section{REFERENCES}

1. Konnickx PR, et al. Suggestive evidence that pelvic endometriosis is a progressive disease, whereas deeply infiltrating endometriosis is associated with pelvic pain. Fertil Steril 1991;55:759-65.

2. Sampson JA. Endometrial carcinoma of the ovary. Arch Surg 1925;10:1.

3. McMeekin DS, Burger RA, et al. Endometrioid adenocarcinoma of the ovary and its relationship to endometriosis. Gynecol Oncol 1995;59:81-86.

4. Ogawa S, et al. Ovarian endometriosis associated with ovarian carcinoma: A clinicopathological and immunohistochemical study. Gyn Oncol 2000;77:298-304.

5. Melin A, Sparen P, Bergqvist A. The risk of cancer and the role of parity among women with endometriosis. Human Repord 2007;22(1):i27-28.

6. Pisarska, Carson SA, Buster JE. Ectopic pregnancy. Lancet 1998;351:1115-20. 